\title{
Quantitative genetic variation of metabolism in the nymphs of the sand cricket, Gryllus firmus, inferred from an analysis of inbred-lines.
}

\author{
ROBERTO F NESPOLO ${ }^{1}$, LUIS E CASTAÑEDA ${ }^{1}$ and DEREK A ROFF 2
}

${ }^{1}$ Instituto de Ecología y Evolución, Facultad de Ciencias, Universidad Austral de Chile, Casilla 567 Valdivia, Chile; ${ }^{2}$ Department of Biology, University of California, Riverside, CA 82521, USA.

\begin{abstract}
Compared with morphological and life history traits, quantitative genetic variation of metabolic and related traits in animals has been poorly studied. We used flow-through $\mathrm{VCO}_{2}$ respirometry and simultaneous activity measurement on nymphs of the sand cricket (Gryllus firmus) from inbred lines to estimate broad-sense heritability of four metabolic variables. In addition, we measured a number of linear dimensions in the adults from the same inbred lines. There were significant multivariate effects of inbred lines for all traits and broadsense heritability for physiological traits was $4.5 \%, 5.2 \%, 10.3 \%$ and $8.5 \%$ for average, resting, minimum and maximum $\mathrm{CO}_{2}$ production in nymphs, respectively. Though the MANOVA indicated significant genetic variation among inbred lines in adult morphology, the broad-sense heritabilities were relatively low ranging from $0-18 \%$. Our results indicate that the heritabilities of metabolic measures are large enough to potentially respond to selection.
\end{abstract}

Key terms: broad-sense heritability, metabolic rate, insects, inbred lines, through-flow $\mathrm{CO}_{2}$ respirometry.

\section{INTRODUCTION}

The potential to respond to natural selection depends on the heritable variation of a trait (Falconer and Mackay, 1997; Lynch and Walsh, 1998). A measure of this variation is heritability, the ratio between the additive genetic and phenotypic variances. Traits can be classified as morphological, physiological, life historical and behavioral (Mousseau and Roff, 1987; Hoffman, 2000). The amount of genetic variation in these types of traits, as well as in different kind of organisms, has been the subject of considerable research and debate (Roff and Mousseau, 1986; Mousseau and Roff, 1987; Price and Schluter, 1991; Houle, 1992; Roff, 1997). Overall, the heritabilities of physiological traits are similar to life history and behavioural traits but lower than morphological traits $(0.31,0.26,0.37$ and 0.51 for physiological, life history, behavioural and morphological traits, respectively. Data from table 3 of Mousseau and Roff [1987]). In Drosophila the heritability of physiological traits appears to be somewhat higher and comparable to morphological traits (Roff and Mousseau, 1986; Hoffman, 2000). However, whereas there are numerous heritability estimates of morphological $(n=140)$ and life history traits $(n=79)$, there is a relative scarcity of heritability estimates for physiological traits $(n=39$, data from table 3 of Mousseau and Roff [1987]). The distribution of estimates is very uneven, with a few animals such as Drosophila, mice, humans and several bird species having been relatively well studied (Mousseau and Roff, 1987), but the majority of species being virtually unknown from any quantitative genetic perspective. Further, physiological traits are not functionally consistent across species. For some animals, many physiological traits (e.g., dessication resistance in insects, 
Hoffman, 2000) are functionally very different than traits classified as "physiological" in other animals (e.g., oxygen carrying capacity by haemoglobin in mammals, Chappell and Snyder, 1984). Hence, it is likely that estimates of the heritability of particular categories of physiological traits cannot be readily used to infer values in other categories. Thus the scarcity of estimates and the breadth of variation within physiology make it difficult to reach any conclusions with respect to the range of heritabilities in this type of trait. For instance, in Drosophila heat resistance, desiccation resistance and starvation are classified as physiological traits but $\mathrm{h}^{2}$ ranges from 0.18 to 0.9 with considerable variation across studies (Hoffman, 2000). Similarly in mammals, body temperature, thermal conductance and basal metabolic rate exhibit a $100 \%$ variation in heritabilities (Nespolo et al., 2003, 2005).

These complications in the analysis of the heritability of physiological traits could be avoided or at least ameliorated by: (1) comparing estimators of different traits obtained from experiments in the same individuals, (2) considering a common physiological trait in very different organisms (e.g. metabolic rate, a general proxy of the "intensity" of functioning of every metacellular animal, represents nearly the same state variable in very different animals). The repeatability of standard metabolic rate is high in several species of insects (Rogowitz and Chappell, 2000; Nespolo et al, 2003a; Terblanche et al, 2004), which is consistent with a high heritability. On the other hand, both broadand narrow-sense heritabilities of metabolic rate appear to be relatively low in vertebrates. For instance, Dohm et al, (2001) presented only negative estimates for Mus, Nespolo et al (2003b) report $\mathrm{h}^{2}=$ 0.14 (non-significant) for basal metabolic rate in a field rodent. The purpose of the experiments reported herein was to determine if the broad-sense heritability of metabolic rate in the sand cricket, Gryllus firmus is consistent with the high repeatabilities estimated in invertebrates or with the low heritabilities reported in vertebrates. Specifically, we used flowthrough $\mathrm{VCO}_{2}$ respirometry to measure the metabolic rates in the nymphs of four highly inbred lines of $G$. firmus. To gain resolution, we extracted several metabolic variables from the $\mathrm{VCO}_{2}$ record, and compared after scaling with morphological variables. Since morphology of adults of these inbred lines is known (Roff and Sokolowska, 2004), we also measured morphological traits as control.

\section{MATERIAL AND METHODS}

\section{Insects}

The inbred lines used in the present experiment were derived from a stock culture that originated from approximately 20 males and 20 females collected in northern Florida in 1981. The stock culture is maintained with a standing adult population of several hundred individuals (generally 100-500 adults, with occasional bottlenecks in which the population may have declined to about 50 adults). To prevent diapause the temperature is maintained in excess of $25^{\circ} \mathrm{C}$. Nymphs and adults in both the stock and the experiment were fed Purina rabbit chow.

The inbred lines were created from the stock population by 14 generations of brother-sister mating, full details of which are given in Roff (2002). After generation 14 the seven extant inbred lines were maintained separately in the same manner as the stock culture. Three inbred lines were lost due to accident leaving four extant lines at the time of the present experiment. As with the original stock, the inbred lines are maintained at population levels of several hundred per cage, with two cages per inbred line. A total of 120 nymphs were drawn haphazardly from the stock cages of the four inbred lines (30 per line), with approximately equal numbers coming from each of two cages per line. Densities within the cages were not controlled and hence heritabilities obtained in the present analysis are likely to be reduced relative to previous analyses in which initial densities were controlled. 


\section{Morphometric measurements}

In all individuals ( 15 adults and 30 nymphs per line), in addition to body mass, we measured head width, head length, prothorax length, and abdomen length. Whereas body mass changes with adult age, all the other measures remain constant after eclosion into the adult morph.

\section{Respirometry}

Our respirometry system was similar to Lighton and Turner (2004) and Rogowitz and Chappell (2000). In brief, carbon dioxide production was measured continuously with an infra-red $\mathrm{CO}_{2}$ analyzer (LI-COR LI6251) capable of resolving differences of 0.2 part per million (ppm) of $\mathrm{CO}_{2}$ in air. The analyser was calibrated periodically against a precision gas mixture (there was almost no drift between calibrations). Flow rates of dry, $\mathrm{CO}_{2}$-free air were maintained at $\pm 1 \%$ by a Sierra mass flow controller. Air was drawn from the ambient, vapour and $\mathrm{CO}_{2}$ water scrubbed with a Drierite-Ascarite column, and flow rate controlled at $95 \mathrm{ml}$ $\mathrm{min}^{-1}$. The metabolic chamber was a $40 \mathrm{ml}$ glass-cylinder. To monitor movement we used a Sable System AD-1 activity detector. Each cricket was measured during 45 minutes but we considered only the last 20 minutes. Each record was automatically transformed by a macro program recorded in the Datacan software (Sable Systems), in order to (1) correct the six-second lag introduced by the distance between the analyzer and the chamber and then to match the activity record with the $\mathrm{VCO}_{2}$ record, and (2) to transform the measure from parts per-million to $\mathrm{ml}-\mathrm{CO}_{2}$ per hour, taking into account the flow rate. For this, we used the standard equation when flowmeter is downstream and $\mathrm{CO} 2$ is scrubbed prior to flow measurement: $\mathrm{VCO} 2=\mathrm{STP} *\left(\mathrm{FeCO}_{2}-\mathrm{FiCO}_{2}\right) * \mathrm{FR} /(1$ - $\left.\mathrm{FeCO}_{2}+\mathrm{FiCO}_{2} / \mathrm{RQ}\right)$, where STP = standard temperature and pressure correction (equal to one with mass-flow controllers), $\mathrm{FeCO} 2=$ excurrent fractional concentration of $\mathrm{CO}_{2}, \mathrm{FiCO}_{2}=$ incurrent fractional concentration of $\mathrm{CO}_{2}, \mathrm{FR}=$ flow rate, $\mathrm{RQ}=$ respiratory quotient, which for herbivorous animals is assumed to be 0.85 .

We took four respirometric variables from each individual: average, resting, maximum and minimum rates. As a "general" measure of metabolism, we took the complete average of each transformed record $\left(=\mathrm{VCO}_{2} \mathrm{avg}\right)$. In addition, we used the activity measurements to detect "resting" periods in each record. Most of the individuals presented a fairly clear pattern of resting and active periods, revealed by the conspicuous drops in $\mathrm{VCO}_{2}$ during periods of undetectable activity (Fig. 1). Resting metabolism ( $\mathrm{VCO}_{2}$ rest) was computed as the average of the one-minute steady state $\mathrm{VCO}_{2}$ consumption during periods of inactivity. In addition, we considered the single maximum $\left(\mathrm{VCO}_{2} \max \right)$ and minimum $\left(\mathrm{VCO}_{2} \mathrm{~min}\right)$ values of $\mathrm{VCO}_{2}$ during the record.

The usual approach for the analysis of $\mathrm{VCO}_{2}$ or $\mathrm{VO}_{2}$ times-series is either to take averages, single values, or a combination of both for parts of whole records. As described above, we followed such procedures but we believe that some information, related to the pattern of peaks and valleys of the record itself is not detected by this procedure. A common approach to capture quantitatively this kind of pattern from time-series is Fast Fourier Transform (FFT). In brief, FFT is used to produce frequency analysis of discrete non-periodic signals. For the case of metabolic time-series, FFT permits adjustment of the peaks and valley structure of the record to a plot of amplitude versus frequency. By this procedure, noisy records (i.e., records with many small peaks of high frequency) produce FFT plots with a low slope (Fig 2 ). Records with a larger variety of peaks (i.e., big peaks and drops, combined with environmental noise) produce a larger (negative) slope of the FFT plot (Fig. 2). Consequently, in addition to the effects of inbred lines on the magnitude of the different measures of $\mathrm{VCO}_{2}$ described above, we examined the variation in the slope of FFT in relation to body mass. 


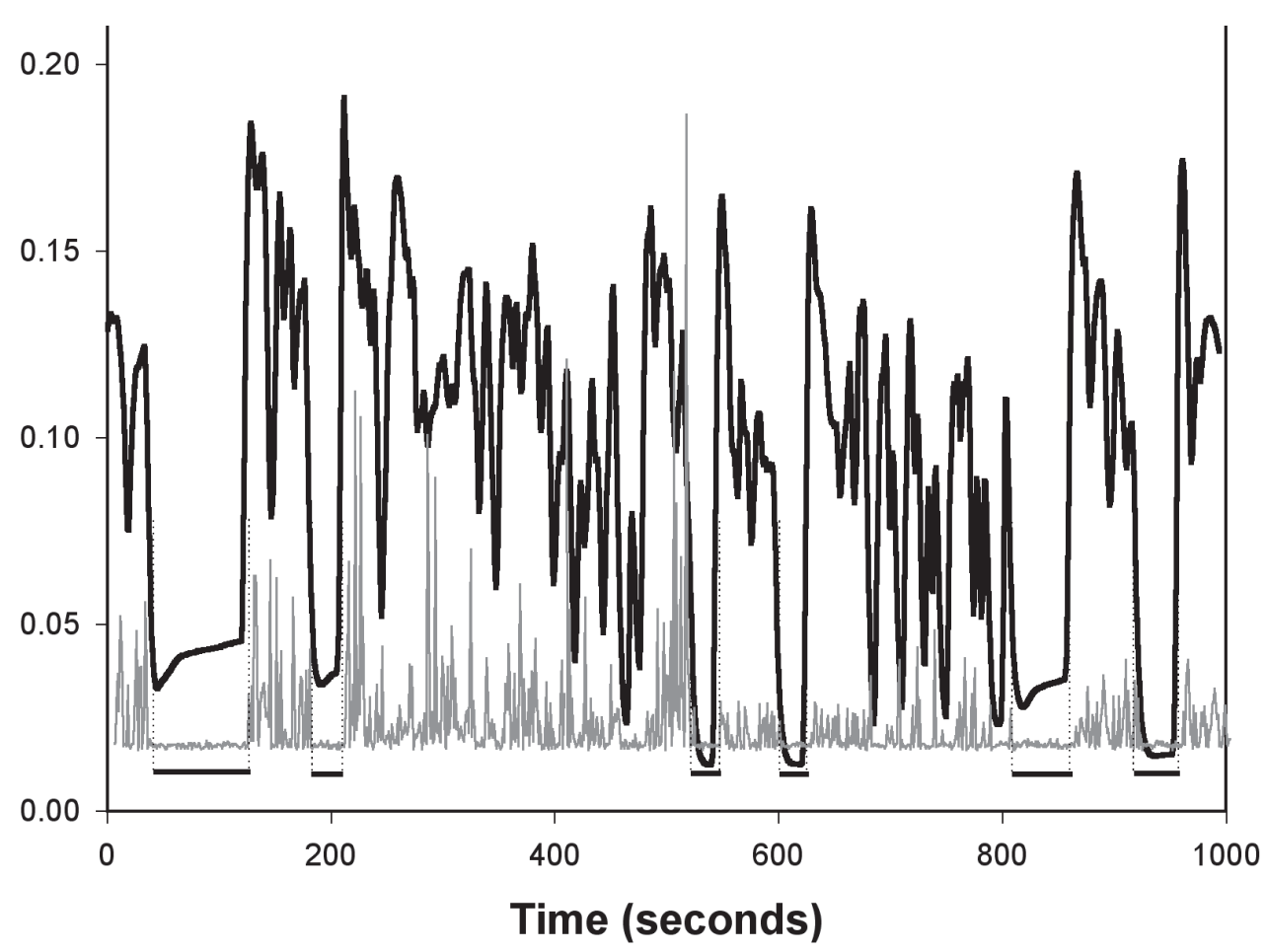

Figure 1: Representative record of $\mathrm{CO}_{2}$ production (black line) and activity measurement (red line, arbitrary units). Black bars at the bottom denote periods of inactivity, evidenced by the match between low activity and low $\mathrm{VCO}_{2}$.

\section{Statistics}

For adults we had two main categorical predictor variables, sex and inbred line, and a single continuous variable, body mass. Because in small nymphs sex cannot be determined, we did not record sex for the nymphs. Broad sense heritabilities were estimated using variance components estimated from a one way analysis of variance. Approximate standard errors for the estimates were computed from the intraclass correlation coefficient variance for full sibs (Falconer and Mackay 1997, pp. 180). Additionally, we tested for overall variation among lines in morphology using MANOVA. We log transformed the metabolic measurements to comply with the assumptions of ANOVA. Heritability estimates were made for the adult morphological measurements but, because the age of the nymphs was unknown and linear measurements change with nymphal age, we did not estimate heritabilities for these traits. However, because body mass is expected to have a large phenotypic effect on metabolic rate, we estimated the heritability of the metabolic variables after correction for body mass effects.

\section{RESULTS}

\section{Adult morphology}

The MANOVA indicated highly significant variation among lines in overall morphology (Wilks $\lambda=0.469, F_{18}, 144.7=2.465, P=$ 0.0016 , Table 1) but no single trait, after the Tukey a-posteriori test, was significantly variable among lines (Table 1). This was also reflected in low broad-sense heritabilities for some traits: $\mathrm{h}^{2}=0,0.02 \pm 0.04,0.05 \pm 0.06$, $0.18 \pm 0.11$, (prothorax length, head width, head length, and prothorax width, respectively). These results indicate that there is genetic variation among lines in morphology, as found previously, but some of the estimations were considerably small (Roff and Sokolovska 2004). 

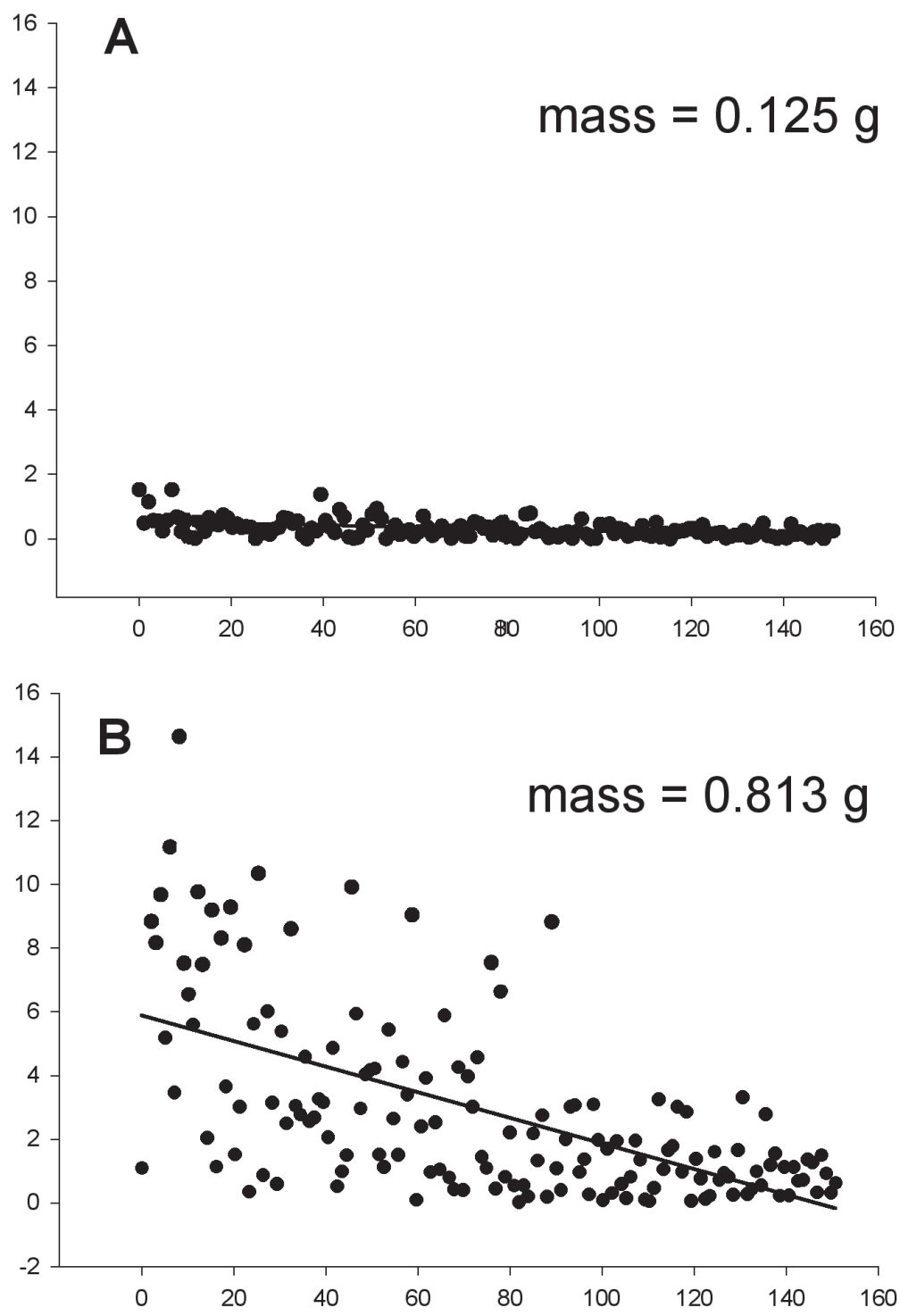

\section{frequency}

Figure 2: Comparison of FFT plots between a very noisy record (A) and a record with a great variety of medium and large peaks (B).

\section{Nymphal metabolic rate}

There were significant effects of inbred lines on the four respirometric variables (Wilks' $\lambda=0.787, \mathrm{~F}_{12,228}=1.8, \mathrm{P}=0.049$, MANCOVA with body mass as a covariate, Table 2). The Tukey post-hoc test, revealed significant differences among inbred lines in all variables (Table
$2)$. The broad-sense heritabilities were $0.045 \pm 0.04$ for $\mathrm{VCO}_{2} \mathrm{avg}, 0.052 \pm 0.06$ for $\mathrm{VCO}_{2}$ rest, $0.10 \pm 0.06$, for $\mathrm{VCO}_{2} \mathrm{~min}$ and $0.085 \pm 0.05$ for $\mathrm{VCO}_{2} \max$. The slope of FFT was significantly correlated with body mass $(\mathrm{r}=0.83 \mathrm{P}=0.001)$ but there were no significant effects attributable to inbred line $\left(\mathrm{F}_{3,115}=1.22 ; \mathrm{P}=0.30\right.$, ANCOVA). 
TABLE 1

Cell statistics (means \pm standard errors) of morphological measurements in adults

\begin{tabular}{lccccc}
\hline & $\mathrm{N}$ & Inbred line 1 & Inbred line 2 & Inbred line 3 & Inbred line 4 \\
\hline Body mass $(\mathrm{g})$ & 15 & $0.681 \pm 0.058$ & $0.832 \pm 0.059$ & $0.924 \pm 0.059$ & $0.743 \pm 0.039$ \\
Head width $(\mathrm{cm})$ & 15 & $0.615 \pm 0.013$ & $0.617 \pm 0.011$ & $0.640 \pm 0.011$ & $0.612 \pm 0.008$ \\
Head length $(\mathrm{cm})$ & 15 & $0.441 \pm 0.014$ & $0.437 \pm 0.014$ & $0.437 \pm 0.018$ & $0.410 \pm 0.013$ \\
Prothorax width $(\mathrm{cm})$ & 15 & $0.688 \pm 0.015$ & $0.684 \pm 0.011$ & $0.728 \pm 0.009$ & $0.688 \pm 0.010$ \\
Prothorax length $(\mathrm{cm})$ & 15 & $0.430 \pm 0.011$ & $0.426 \pm 0.011$ & $0.432 \pm 0.012$ & $0.429 \pm 0.010$ \\
Abdomen width $(\mathrm{cm})$ & 15 & $0.738 \pm 0.027$ & $0.770 \pm 0.032$ & $0.763 \pm 0.024$ & $0.709 \pm 0.020$ \\
Abdomen length $(\mathrm{cm})$ & 15 & $1.574 \pm 0.064$ & $1.615 \pm 0.050$ & $1.767 \pm 0.050$ & $1.541 \pm 0.044$ \\
\hline
\end{tabular}

TABLE 2

Cell statistics (means \pm standard errors, $\mathrm{N}$ in parentheses) of morphological and physiological measurements in nymphs. Sample sizes differ since not all individuals could be measured because of noisy records and/or tissue damage during manipulation

\begin{tabular}{lcccc}
\hline & Inbred line 1 & Inbred line 2 & Inbred line 3 & Inbred line 4 \\
\hline $\mathrm{VCO}_{2} \mathrm{avg}\left(\mathrm{mlCO}_{2} \mathrm{~h}^{-1}\right)$ & $(30) 0.093 \pm 0.011$ & $(30) 0.088 \pm 0.015$ & (30) $0.132 \pm 0.018$ & (30) $0.093 \pm 0.012$ \\
$\mathrm{VCO}_{2} \min \left(\mathrm{mlCO}_{2} \mathrm{~h}^{-1}\right)$ & $(29) 0.036 \pm 0.005$ & $(30) 0.029 \pm 0.004$ & $(30) 0.056 \pm 0.011$ & (30) $0.020 \pm 0.003$ \\
$\mathrm{VCO}_{2} \mathrm{max}\left(\mathrm{mlCO}_{2} \mathrm{~h}^{-1}\right)$ & $(30) 0.158 \pm 0.017$ & $(30) 0.137 \pm 0.022$ & (30) $0.221 \pm 0.032$ & (30) $0.148 \pm 0.016$ \\
$\mathrm{VCO}_{2} \mathrm{rest}\left(\mathrm{mlCO}_{2} \mathrm{~h}^{-1}\right)$ & $(22) 0.062 \pm 0.010$ & $(24) 0.044 \pm 0.007$ & $(24) 0.084 \pm 0.016$ & (24) $0.040 \pm 0.008$ \\
FFTslope & $(30) 0.020 \pm 0.003$ & (30) $0.018 \pm 0.003$ & (30) $0.025 \pm 0.004$ & (30) $0.021 \pm 0.003$ \\
\hline
\end{tabular}

\section{DISCUSSION}

One of the least known aspects of insect physiology is the genetic variance of physiological traits, especially metabolic rate (Hoffmann, 2000; Nespolo et al, 2003a). This is rather surprising as flowthrough respirometry has been intensely used to study a very wide range of factors correlated with metabolic rate, such as age (Hack, 1997), diet composition (Zanotto et $a l, 1997)$, reproductive cost (Prestwich and Walker, 1981; Hack, 1997, 1998), flight and locomotion (Rogowitz and Chappell, 2000), and adaptive features such as seasonal acclimation (Forlow and MacMahon, 1988), dry habitat adaptations (Cooper, 1993), altitude (Hadley and Massion, 1985), latitude across populations (Rourke, 2000) and species differences (Davis et al, 2003). All these patterns of physiological adaptation are potential consequences of past genetic variation in metabolic rate, making genetic variance a very important quantity. But, so far as we are aware, no study has addressed the heritability of metabolic rate. Repeatability studies suggest that metabolic rate is consistent over time in insects, and is consistent with a high heritability (Nespolo et al, 2003a; Chappell and Rogowitz, 2000; Terblanche et al 2004). The low broad sense heritabilities we found in morphological traits of adults $(0-0.18)$ are lower than obtained in previous studies in adults of the seven inbred stocks, (Roff and Sokolovska 2004). This fact could be in part explained by the reduction sample size and because of the absence of density control in the cages (see methods). It is known that rearing conditions such as density and temperature could affect adult morphology in insects (Begin et al., 2004). This effect in cages would inflate the 
environmental variance thus reducing computed heritability. Nevertheless, the MANOVA did demonstrate genetic variation for overall adult morphology among the four inbred lines. Given the low variance in the morphological traits, the heritabilities of metabolic rate measures (0.04-0.10) are perhaps underestimated, but this observation is only qualitative. For the case of FFT slopes (i.e., the pattern of the metabolic time-series), no genetic effects were detected, but a strong body mass dependence. The biological interpretation of this finding is that larger individuals exhibit also larger peaks and valleys than small ones, which exhibit more continuous patterns. These bursts of $\mathrm{CO}_{2}$ in large crickets could be due to some form of cyclic gas exchange, such as in carabid beetles and moth pupae (Chown et al., 2006). However, this needs to be confirmed since no cricket has exhibited DGC to date.

Overall, these data show that metabolic rate is an "evolvable" trait within populations, but further experiments are required to determine how much of the variance is due to additive effects. This picture is somewhat inconsistent with the information available from other animals: mice and wild rodents appear to have a very low heritability of metabolic rate (negative estimate in Dohm et al 2001; 0.14 in Nespolo et al 2003b; 0.01 in Bacigalupe et al 2004). Also, physiological traits other than metabolic rate (i.e., heat and cold resistance, ethanol resistance and dissecation resistance) in Drosophila present narrow-sense heritabilities below 0.2 (Hoffman, 2000). However, as we advanced in the introduction, physiology is a very heterogeneous category and generalizations within this category may be misleading. The difficulties of measuring metabolic rate in animals have impeded accurate estimates of heritabilities, as reflected in generally low sample sizes: for example, the cited studies above were all performed using sample sizes of less than 26 families (Dohm et al 2001; Nespolo et al 2003b; Bacigalupe et al 2004). Estimates of heritabilities of metabolism in mammals obtained with larger sample sizes appear to give large and significant narrow-sense heritabilities (Nespolo et al., unpublished results; Koteja, personal communication).

The existence of genetic variation in metabolism in the nymphs of the sand cricket suggests that selection over energy processing capacities would generate an evolutionary response (although it will depend on the magnitude of the selection differential). The implications of this are important for several reasons. Energy consumption during larval period is a proxy for energy processing efficiency (Gouveia et al 2000), which in turn determines growth rate and possibly adult size. Given the tradeoff between growth rate and reproduction (Roff, 2000), it would be reasonable to hypothesize that the metabolic rate of the nymphs could be a determinant of the reproductive output of adults (eg., larger females could produce more eggs and larger males could have an advantage in time spent calling and hence attracting females, Roff, 2000). An alternative scenario is an energetic trade-off, with a higher metabolic rate reducing the energy allocated to growth. In adult crickets, energetic trade-offs have been observed in the cost of wing maintenance, where macropters due to their massive flight muscles have a higher metabolic rate than micropters, and divert less energy to gonads (affecting fecundity) (Crnokrak and Roff, 2002). Such a trade-off could not occur in the nymphs as they do not have wings and mature gonads, but metabolic rates in the nymphs may translate into constraints in the adults via genetic correlations between metabolic rates at different ages. The important finding of the present study is that metabolism (or energetic efficiency) is genetically variable and hence can be a target of natural selection.

\section{ACKNOWLEDGEMENTS}

This work was partially supported by a MECESUP fellowship and Fondecyt grant No 3030032 to Roberto Nespolo, and funds supplied to DAR by an initial complement grant from UCR, to Derek Roff. Luis Castañeda acknowledges a Conicyt fellowship. 


\section{REFERENCES}

BACIGALUPE LD, NESPOLO RF, BUSTAMANTE DM, BOZINOVIC F (2004) The quantitative genetics of sustained energy budget in a wild mouse. Evolution 58: 421-429

CHAPPELL MA, SNYDER LRG (1984) Biochemical and physiological correlates of deer mouse alpha-chain hemoglobin polymorphisms. Proc.Natl.Acad.Sci. 81: 5484-548

CHAPPELL MA, ROGOWITZ GL (2000) Mass, temperature and metabolic effects on discontinuous gas exchange cycles in eucalyptus-boring beetles (Coleoptera: Creambycidae). Journal of Experimental Biology 203: 3809-3820

CHOWN SL, GIBBS AG, HETZ SK, KLOK CJ, LIGHTON JRB,, MARAIS E. (2006) Discontinuous gas exchange in insects: a clarification of hypotheses and approaches. Physiological and Biochemical Zoology 79: 333-339

COOPER PD (1993) Field metabolic rate and cost of activity in two tenebrionid beetles from the Mojave desert of North America. Journal of Arid Environments 24: $165-175$

CRNOKRAK P, ROFF DA (2002) Trade-offs to flight capability in Gryllus firmus: the influence of wholeorganism respiration rate on fitness. Journal of Evolutionary Biology 15: 388-398

DAVIS ALV, CHOWN SL, MCGEOCH MA, SCHOLTZ $\mathrm{CH}$ (2003) A comparative analysis of metabolic rate in six Scarabaeus species (Coleoptera: Scarabaeidae) from southern Africa: further caveats when inferring adaptation. Journal of Insect Physiology 46: 553-562

DOHM MR, HAYES JP, GARLAND T (2001) The quantitative genetics of maximal and basal rates of oxygen consumption in mice. Genetics 159: 267-277

FALCONER DS, MACKAY TFC (1997) Introduction to Quantitative Genetics. Longman, Edimburgh

FORLOW LJ, MACMAHON JA (1988) A seasonal comparison of metabolic and water loss rates of three species of grasshoppers. Comparative Biochemistry and Physiology 89A: 51-60

GOUVEIA SM, SIMPSON SJ, RAUBENHEIMER D, ZANOTTO FP (2000) Patterns of respiration in Locusta migratoria nymphs when feeding . Physiological Entomology 25: 88-93

HACK MA (1997) The effects of mass and age on standard metabolic rate in house crickets. Physiological Entomology 22: 325-331

HACK MA (1998) The energetics of male mating strategies in field crickets (Orthoptera: Gryllinae: Gryllidae). Journal of Insect Behavior 11: 853-867

HADLEY NF, MASSION DD (1985) Oxygen consumption, water loss and cuticular lipids of high and low elevation populations of the grasshopper Aeropedellus clavatus (Prthoptera: Acrididae). Comparative Biochemistry and Physiology 80A: 307-311

HOFFMAN AA (2000) Laboratory and field heritabilities. Some lessons from Drosophila. In: Mousseau TA, Sinervo B and Endler J (eds) Adaptive Genetic Variation in the Wild, pp 200-218. Oxford University Press, New York

HOULE D (1992) Comparing evolvability and variability of quantitative traits. Genetics 130: 195-204
LIGHTON JRB, R TURNER (2004) Thermolimit respirometry: an objective assessment of critical thermal maxima in two desert harvester ants, Pogonomyrmex rugosus and P. californicus. Journal of Experimental Biology 207: 1903-1913

LYNCH M, WALSH B (1998) Genetics and Analysis of Quantitative Traits. Sinauer, Sunderland

MOUSSEAU TA, ROFF DA (1987) Natural selection and the heritability of fitness components. Heredity 59: 181-197

NESPOLO RF, LARDIES MA, BOZINOVIC F (2003a) Intrtapopulational variation in the standard metabolic rate of insects: repeatability, thermal dependence and sensitivity (Q10) of oxygen consumption in a cricket. Journal of Experimental Biology 206: 4309-4315

NESPOLO RF, BACIGALUPE LD, BOZINOVIC F (2003b) Heritability of energetics in a wild mammal, the leaf-eared mouse (Phyllotis darwini). Evolution 57: $1679-1688$

NESPOLO RF, BUSTAMANTE DM, BACIGALUPE LD, BOZINOVIC F (2005) Quantitative genetics of bioenergetics and growth-related traits in the wild mammal, Phyllotis darwini. Evolution 59: 1829-1837

PRICE TD, SCHLUTER D (1991) On the low heritability of life history traits. Evolution 45: 853-861

PRESTWICH KN, WALKER TJ (1981) Energetics of singing in crickets: effect of temperature in three trilling species (Orthoptera: Gryllidae). Oecologia 143: 199-212

ROFF DA, MOUSSEAU TA (1986) Quantitative genetics and fitness: lessons from Drosophila. Heredity 58: 103-118

ROFF DA (1997) Evolutionary Quantitative Genetics. Chapman \& Hall, Montreal

ROFF DA (2000) Trade-offs between growth and reproduction: an analysis of the quantitative genetic evidence. Journal of Evolutionary Biology 13: 434-445

ROFF DA (2002) Inbreeding depression: tests of the overdominance and partial dominance hypothesis. Evolution 56: 768-775

ROFF, DA, RÉALE D (2004) The quantitative genetics of fluctuating asymmetry: a comparison of two models. Evolution 58: 47-58

ROFF DA, SOKOLOVSKA N (2004) Extra-nuclear effects on growth and development in the sand cricket Gryllus firmus. Journal of Evolutionary Biology 17: 663-671

ROGOWITZ GL, CHAPPELL MA (2000) Energy metabolism of eucalyptus-boring beetles at rest and during locomotion: gender makes a difference. Journal of Experimental Biology 203: 1131-1139

ROURKE B (2000) Geographic and altitudinal variation in water balance and metabolic rate in a California grasshopper, Melanoplus sanguinipes. Journal of Experimental Biology 203: 2699-2712

TERBLANCHE JS, KLOK CJ, CHOWN SL (2004) Metabolic rate variation in Glossina pallidipes (Diptera: Glossinidae): gender, ageing and repeatability. Journal of Insect Physiology 50: 419-428

ZANOTTO FP, GOUVEIA SM, SIMPSON SJ, RAUBENHEIMER D, CALDER PC (1997) Nutrititional homeostasis in locusts: is there a mechanism for increased energy expenditure during carbohydrate overfeeding? Journal of Experimental Biology 200: 2437-2448 